\title{
A version of Putinar's Positivstellensatz for cylinders
}

\author{
Paula Escorcielo* Daniel Perrucci* \\ Departamento de Matemática, FCEN, Universidad de Buenos Aires, Argentina \\ IMAS, CONICET-UBA, Argentina
}

\begin{abstract}
We prove that, under some additional assumption, Putinar's Positivstellensatz holds on cylinders of type $S \times \mathbb{R}$ with $S=\left\{\bar{x} \in \mathbb{R}^{n} \mid g_{1}(\bar{x}) \geq 0, \ldots, g_{s}(\bar{x}) \geq 0\right\}$ such that the quadratic module generated by $g_{1}, \ldots, g_{s}$ in $\mathbb{R}\left[X_{1}, \ldots, X_{n}\right]$ is archimedean, and we provide a degree bound for the representation of a polynomial $f \in \mathbb{R}\left[X_{1}, \ldots, X_{n}, Y\right]$ which is positive on $S \times \mathbb{R}$ as an explicit element of the quadratic module generated by $g_{1}, \ldots, g_{s}$ in $\mathbb{R}\left[X_{1}, \ldots, X_{n}, Y\right]$. We also include an example to show that an additional assumption is necessary for Putinar's Positivstellensatz to hold on cylinders of this type.
\end{abstract}

\section{Introduction}

Putinar's Positivstellensatz ([1] $)$ is one of the most celebrated results in the theory of sums of squares and certificates of non-negativity. This theorem states that given $g_{1}, \ldots, g_{s} \in \mathbb{R}[\bar{X}]=\mathbb{R}\left[X_{1}, \ldots, X_{n}\right]$ such that the quadratic module $M\left(g_{1}, \ldots, g_{s}\right)$ generated by $g_{1}, \ldots, g_{s}$ in $\mathbb{R}[\bar{X}]$ is archimedean, every $f \in \mathbb{R}[\bar{X}]$ positive on

$$
S=\left\{\bar{x} \in \mathbb{R}^{n} \mid g_{1}(\bar{x}) \geq 0, \ldots, g_{s}(\bar{x}) \geq 0\right\}
$$

belongs to $M\left(g_{1}, \ldots, g_{s}\right)$, which is a certificate of the non-negativity of $f$.

We explain now the terminology in the preceding paragraph. A subset $M \subset \mathbb{R}[\bar{X}]$ is a quadratic module if it satisfies

- $1 \in M$,

- $M+M \subset M$,

- $\mathbb{R}[\bar{X}]^{2} M \subset M$ (i.e. $M$ is closed under multiplication by squares).

The set of sums of squares $\sum \mathbb{R}[\bar{X}]^{2}$ is the smallest quadratic module in $\mathbb{R}[\bar{X}]$. Given $g_{1}, \ldots, g_{s} \in \mathbb{R}[\bar{X}]$, the quadratic module generated by these polynomials in $\mathbb{R}[\bar{X}]$ is

$$
M\left(g_{1}, \ldots, g_{s}\right)=\left\{\sigma_{0}+\sigma_{1} g_{1}+\cdots+\sigma_{s} g_{s} \mid \sigma_{0}, \sigma_{1}, \ldots, \sigma_{s} \in \sum \mathbb{R}[\bar{X}]^{2}\right\}
$$

*Partially supported by the Argentinian grants UBACYT 20020160100039BA and PIP 11220130100527CO CONICET. MSC Classification: 12D15, 13J30, 14P10.

Keywords: Putinar's Positivstellenatz, Sums of squares, Degree bounds. 
and it is the smallest quadratic module in $\mathbb{R}[\bar{X}]$ which contains $g_{1}, \ldots, g_{s}$. Every polynomial $f \in$ $M\left(g_{1}, \ldots, g_{s}\right)$ is non-negative on the set $S \subset \mathbb{R}^{n}$ but the converse is not true in general (see [14, Example]). A quadratic module $M$ in $\mathbb{R}[\bar{X}]$ is said to be archimedean if there exists $N \in \mathbb{R}_{>0}$ such that

$$
N-X_{1}^{2}-\cdots-X_{n}^{2} \in M
$$

If the quadratic module $M\left(g_{1}, \ldots, g_{s}\right)$ is archimedean, then the set $S \subset \mathbb{R}^{n}$ is compact, but again, the converse is not true in general (see [3, Example 4.6]).

Another of the most important results in the theory of sums of squares and certificates of non-negativity is Schmüdgen's Positivstellensatz ([12]). This theorem states that given $g_{1}, \ldots, g_{s} \in \mathbb{R}[\bar{X}]$ such that the set $S \subset \mathbb{R}^{n}$ is compact, every $f \in \mathbb{R}[\bar{X}]$ positive on $S$ belongs to the preordering $T\left(g_{1}, \ldots, g_{s}\right)$ generated by $g_{1}, \ldots, g_{s}$ in $\mathbb{R}[\bar{X}]$, which is a certificate of the non-negativity of $f$.

As before, we explain the terminology we have just used. A subset $T \subset \mathbb{R}[\bar{X}]$ is a preordering if it satisfies

- $\mathbb{R}[\bar{X}]^{2} \subset T$,

- $T+T \subset T$,

- $T T \subset T$ (i.e. $T$ is closed under multiplication).

The set of sums of squares $\sum \mathbb{R}[\bar{X}]^{2}$ is the smallest preordering in $\mathbb{R}[\bar{X}]$. It is easy to see that $T \subset \mathbb{R}[\bar{X}]$ is a preordering if and only if it is a quadratic module and it is closed under multiplication. Given $g_{1}, \ldots, g_{s} \in \mathbb{R}[\bar{X}]$, the preordering generated by these polynomials in $\mathbb{R}[\bar{X}]$ is

$$
T\left(g_{1}, \ldots, g_{s}\right)=\left\{\sum_{I \subset\{1, \ldots, s\}} \sigma_{I} \prod_{i \in I} g_{i} \mid \sigma_{I} \in \sum \mathbb{R}[\bar{X}]^{2} \text { for every } I \subset\{1, \ldots, s\}\right\}
$$

and it is the smallest preordering in $\mathbb{R}[\bar{X}]$ which contains $g_{1}, \ldots, g_{s}$. It is clear that $M\left(g_{1}, \ldots, g_{s}\right)$ is included in $T\left(g_{1}, \ldots, g_{s}\right)$. Every polynomial $f \in T\left(g_{1}, \ldots, g_{s}\right)$ is non-negative on the set $S \subset \mathbb{R}^{n}$, but the converse is not true in general (again, see [14, Example]).

Both Schmüdgen's Positivstellensatz and Putinar's Positivstellensatz provide a representation of a polynomial on a basic closed semialgebraic set which makes evident the non-negativity of the polynomial. A natural question is if it is possible to bound the degrees of all the different terms in these representations. Answers to this question have been given by Schweighofer ([13, Theorem 3]) in the case of Schmüdgen's Positivstellensatz and by Nie and Schweighofer ([8, Theorem 6]) in the case of Putinar's Positivstellensatz. In the particular case where $S$ is the hypercube $[0,1]^{n}$, improved bounds have been given in [2] and [6]. We include here the precise statement of [8, Theorem 6], but we introduce first some useful definition already present in [8, [9], 10], 13], etc.

\section{Definition 1 For}

$$
f=\sum_{\substack{\alpha \in \mathbb{N}_{0}^{n} \\
|\alpha| \leq d}}\left(\begin{array}{c}
|\alpha| \\
\alpha
\end{array}\right) a_{\alpha} \bar{X}^{\alpha} \in \mathbb{R}[\bar{X}]
$$


we consider the norm of $f$ defined by

$$
\|f\|=\max \left\{\left|a_{\alpha}\right|\left|\alpha \in \mathbb{N}_{0}^{n},\right| \alpha \mid \leq d\right\}
$$

where for $\alpha=\left(\alpha_{1}, \ldots, \alpha_{n}\right) \in \mathbb{N}_{0}^{n}$,

$$
|\alpha|=\alpha_{1}+\cdots+\alpha_{n} \quad \text { and } \quad\left(\begin{array}{c}
|\alpha| \\
\alpha
\end{array}\right)=\frac{|\alpha| !}{\alpha_{1} ! \ldots \alpha_{n} !} .
$$

Note that the definition of this norm is made in such a way that for every $d \in \mathbb{N},\left\|\left(X_{1}+\cdots+X_{n}\right)^{d}\right\|=1$. The precise statement of [8, Theorem 6] is the following.

Theorem 2 (Putinar's Positivstellensatz with degree bound) Let $g_{1}, \ldots, g_{s} \in \mathbb{R}[\bar{X}]$ such that

$$
\emptyset \neq S=\left\{\bar{x} \in \mathbb{R}^{n} \mid g_{1}(\bar{x}) \geq 0, \ldots, g_{s}(\bar{x}) \geq 0\right\} \subset(-1,1)^{n}
$$

and such that the quadratic module $M\left(g_{1}, \ldots, g_{s}\right)$ is archimedean. There exists a positive constant $c$ such that for every $f \in \mathbb{R}[\bar{X}]$ positive on $S$, if $\operatorname{deg} f=d$ and $\min \{f(\bar{x}) \mid \bar{x} \in S\}=f^{*}>0, f$ can be written as

$$
f=\sigma_{0}+\sigma_{1} g_{1}+\cdots+\sigma_{s} g_{s} \in M\left(g_{1}, \ldots, g_{s}\right)
$$

with $\sigma_{0}, \sigma_{1}, \ldots, \sigma_{s} \in \sum \mathbb{R}[\bar{X}]^{2}$ and

$$
\operatorname{deg}\left(\sigma_{0}\right), \operatorname{deg}\left(\sigma_{1} g_{1}\right), \ldots, \operatorname{deg}\left(\sigma_{s} g_{s}\right) \leq c \mathrm{e}^{\left(\frac{\|f\| d^{2} n^{d}}{f^{*}}\right)^{c}} .
$$

In the degree bound above, $\mathrm{e}=2.718 \ldots$ is the base of the natural logarithm. Note that the constant $c$ depends on $g_{1}, \ldots, g_{s}$ but it is independent of $f$.

The problem of representing positive polynomials as sums of squares for cylinders with compact crosssection has been studied within the more general framework of the moment problem in [4], [5] and [9]. Under some extra mild assumption, in [9, Theorem 3] Powers obtains an extension of Schmüdgen's Positivstellensatz to cylinders of type $S \times F$ with $S \subset \mathbb{R}^{n}$ a compact semialgebraic set and $F \subset \mathbb{R}$ an unbounded closed semialgebraic set. The precise extra assumption under consideration is the following.

Definition 3 Let $f \in \mathbb{R}[\bar{X}, Y], m=\operatorname{deg}_{Y} f$ and $S \subset \mathbb{R}^{n}$. The polynomial $f$ is fully $m$-ic on $S$ if for every $\bar{x} \in S, f(\bar{x}, Y) \in \mathbb{R}[Y]$ has degree $m$.

In other words, the condition of being fully $m$-ic on $S$ is that, when the variable $Y$ is distinguished, the leading coefficient (which is a polynomial in $\mathbb{R}[\bar{X}]$ ) does not vanish on $S$. To obtain [9, Theorem 3], given $f \in \mathbb{R}[\bar{X}, Y]$ a positive polynomial on $S \times F$, the idea is to consider the variable $Y$ as a parameter and to produce a uniform version of [13, Theorem 3], in such a way that all the representations obtained for all the specializations of $Y$ can be glued together to obtain the desired representation for $f$.

In this paper we borrow and combine many ideas and techniques from [8], 9] and [13] to extend Putinar's Positivstellensatz to cylinders of type $S \times \mathbb{R}$, again under the extra assumption in Definition 3. Before stating our main result, we introduce some definition and notation. 


\section{Definition 4 For}

$$
f=\sum_{0 \leq i \leq m} \sum_{\substack{\alpha \in \mathbb{N}_{0}^{n} \\
|\alpha| \leq d}}\left(\begin{array}{c}
|\alpha| \\
\alpha
\end{array}\right) a_{\alpha, i} \bar{X}^{\alpha} Y^{i} \in \mathbb{R}[\bar{X}, Y]
$$

we consider another norm of $f$ defined by

$$
\|f\|_{\bullet}=\max \left\{\left|a_{\alpha, i}\right|\left|0 \leq i \leq m, \alpha \in \mathbb{N}_{0}^{n},\right| \alpha \mid \leq d\right\} .
$$

Notation 5 For

$$
f=\sum_{0 \leq i \leq m} f_{i}(\bar{X}) Y^{i} \in \mathbb{R}[\bar{X}, Y]
$$

with $f_{m} \neq 0$, we note by

$$
\bar{f}=\sum_{0 \leq i \leq m} f_{i}(\bar{X}) Y^{i} Z^{m-i} \in \mathbb{R}[\bar{X}, Y, Z]
$$

its homogenization with respect to the variable $Y$.

Let $\emptyset \neq S \subset \mathbb{R}^{n}$ be a compact set and let $f \in \mathbb{R}[\bar{X}, Y]$. If $f$ is fully $m$-ic on $S$ and $f>0$ on $S \times \mathbb{R}$, it is clear that $m$ is even and $f_{m}>0$ on $S$. It can also be easily seen that if we take

$$
C=\left\{(y, z) \in \mathbb{R}^{2}, y^{2}+z^{2}=1\right\}
$$

then $\bar{f}>0$ on the compact set $S \times C$.

Notation 6 For $g_{1}, \ldots, g_{s} \in \mathbb{R}[\bar{X}]$, we denote

$$
M_{\mathbb{R}[\bar{X}, Y]}\left(g_{1}, \ldots, g_{s}\right)=\left\{\sigma_{0}+\sigma_{1} g_{1}+\cdots+\sigma_{s} g_{s} \mid \sigma_{0}, \sigma_{1}, \ldots, \sigma_{s} \in \sum \mathbb{R}[\bar{X}, Y]^{2}\right\}
$$

the quadratic module generated by $g_{1}, \ldots, g_{s}$ in $\mathbb{R}[\bar{X}, Y]$.

Note that we keep the notation $M\left(g_{1}, \ldots, g_{s}\right)$ for the quadratic module generated by $g_{1}, \ldots, g_{s}$ in $\mathbb{R}[\bar{X}]$. We state now our main theorem.

Theorem 7 Let $g_{1}, \ldots, g_{s} \in \mathbb{R}[\bar{X}]$ such that

$$
\emptyset \neq S=\left\{\bar{x} \in \mathbb{R}^{n} \mid g_{1}(\bar{x}) \geq 0, \ldots, g_{s}(\bar{x}) \geq 0\right\} \subset(-1,1)^{n}
$$

and such that the quadratic module $M\left(g_{1}, \ldots, g_{s}\right)$ is archimedean. There exists a positive constant $c$ such that for every $f \in \mathbb{R}[\bar{X}, Y]$ positive on $S \times \mathbb{R}$, if $\operatorname{deg}_{\bar{X}} f=d, \operatorname{deg}_{Y} f=m$ with $f$ fully $m$-ic on $S$ and

$$
\min \{\bar{f}(\bar{x}, y, z) \mid \bar{x} \in S,(y, z) \in C\}=f^{\bullet}>0
$$

$f$ can be written as

$$
f=\sigma_{0}+\sigma_{1} g_{1}+\cdots+\sigma_{s} g_{s} \in M_{\mathbb{R}[\bar{X}, Y]}\left(g_{1}, \ldots, g_{s}\right)
$$

with $\sigma_{0}, \sigma_{1}, \ldots, \sigma_{s} \in \sum \mathbb{R}[\bar{X}, Y]^{2}$ and

$$
\operatorname{deg}\left(\sigma_{0}\right), \operatorname{deg}\left(\sigma_{1} g_{1}\right), \ldots, \operatorname{deg}\left(\sigma_{s} g_{s}\right) \leq c(m+1) 2^{\frac{m}{2}} \mathrm{e}^{\left(\frac{\|f\| \bullet(m+1) d^{2}(3 n)^{d}}{f^{\bullet}}\right)^{c}} .
$$


As in Theorem 2, the constant $c$ depends on $g_{1}, \ldots, g_{s}$ but it is independent of $f$. Note that, when $m=0$, this is to say, $f \in \mathbb{R}[\bar{X}]$, the bound in Theorem 7 is of similar type to the bound in Theorem 2. Actually, in Remark 21 we see that if $n \geq 2$, the factor $3^{d}$ in the exponent can be hidden in the constant $c$ and therefore the bound in Theorem 7 is of the same type to the bound in Theorem 2 .

Theorem 7 is basically Putinar's Positivstellensatz under the additional assumption that $f$ is fully $m$-ic on $S$. Next example, which is a variation of [14, Example] shows that either this one or some other additional assumption is indeed necessary.

Example 8 Take $g_{1}=\left(1-X^{2}\right)^{3} \in \mathbb{R}[X]$, then $S=[-1,1] \subset \mathbb{R}$ and $M\left(g_{1}\right)$ is archimedean since

$$
\frac{4}{3}-X^{2}=\frac{4}{3} X^{2}\left(X^{2}-\frac{3}{2}\right)^{2}+\frac{4}{3}\left(1-X^{2}\right)^{3} .
$$

Now take $f(X, Y)=\left(1-X^{2}\right) Y^{2}+1 \in \mathbb{R}[X, Y]$. It is clear that $f>0$ in $S \times \mathbb{R}$ but $f$ is not fully 2 -ic on $S$. If $f \in M_{\mathbb{R}[X, Y]}\left(g_{1}\right)$, we have an identity

$$
\left(1-X^{2}\right) Y^{2}+1=\sum_{1 \leq j \leq s}\left(\sum_{0 \leq i \leq m^{\prime}} p_{j i}(X) Y^{i}\right)^{2}+\sum_{1 \leq j \leq s}\left(\sum_{0 \leq i \leq m^{\prime}} q_{j i}(X) Y^{i}\right)^{2}\left(1-X^{2}\right)^{3}
$$

with at least one of $p_{1 m^{\prime}}, \ldots, p_{s m^{\prime}}, q_{1 m^{\prime}}, \ldots, q_{s m^{\prime}}$ not identically zero. Looking at the degree in $Y$ at both sides of (11), we have $m^{\prime} \geq 1$.

If $m^{\prime} \geq 2$, looking at the terms of degree $2 m^{\prime}$ in $Y$ at both sides of (11) we have

$$
0=\sum_{1 \leq j \leq s} p_{j m^{\prime}}(X)^{2}+\sum_{1 \leq j \leq s} q_{j m^{\prime}}(X)^{2}\left(1-X^{2}\right)^{3}
$$

but this is impossible since the polynomial on the right hand side is positive in $[-1,1]$ with the only possible exception of a finite number of points. Indeed, any point in $(-1,1)$ such that the polynomial on the right hand side vanishes at, should be a common root of $p_{1 m^{\prime}}, \ldots, p_{s m^{\prime}}, q_{1 m^{\prime}}, \ldots, q_{s m^{\prime}}$; but at least one of these polynomials is not identically zero and therefore has a finite number of roots.

If $m^{\prime}=1$, looking at the terms of degree 2 in $Y$ at both sides of (1) we have

$$
1-X^{2}=\sum_{1 \leq j \leq s_{1}} p_{j 1}(X)^{2}+\sum_{1 \leq j \leq s_{2}} q_{j 1}(X)^{2}\left(1-X^{2}\right)^{3} \in M\left(g_{1}\right)
$$

and this is impossible since it is exactly the well-known example from [14, Example].

As in [9, Theorem 3], the general idea to prove Theorem 7 is, given $f \in \mathbb{R}[\bar{X}, Y]$ a positive polynomial on $S \times \mathbb{R}$, to consider the variable $Y$ as a parameter and to produce, this time, a uniform version of Theorem 2 ([8, Theorem 6$])$, in such a way that all the representations obtained for all the specializations of $Y$ can be glued together to obtain the desired representation for $f$.

Actually, the proof of [8, Theorem 6] uses [13, Theorem 3], and the proof of [13, Theorem 3] uses the bound for Pólya's Theorem from [10, Theorem 1]. For us, in order to succeed to prove Theorem 7 following the described strategy, we need to reorganize these ideas in a way that we use directly [10, Theorem 1] without going through [13, Theorem 3] as a packaged theorem (even though we use ideas from its proof). 
Since Pólya's Theorem plays such a significant role, we first prove the following auxiliary proposition, which is a version of Theorem 7 under the extra assumption that the set $S$ is included in the interior of a convenient simplex. Then, Theorem 7 is obtained by simply composing with a linear change of variables.

Notation 9 For $n \in \mathbb{N}$, we denote by $\widetilde{\Delta}_{n}$ the simplex

$$
\widetilde{\Delta}_{n}=\left\{\bar{x} \in \mathbb{R}^{n} \mid \sum_{1 \leq i \leq n} x_{i} \leq 1 \text { and } x_{i} \geq 0 \text { for } 1 \leq i \leq n\right\} \text {. }
$$

Proposition 10 Let $g_{1}, \ldots, g_{s} \in \mathbb{R}[\bar{X}]$ such that

$$
\emptyset \neq S=\left\{\bar{x} \in \mathbb{R}^{n} \mid g_{1}(\bar{x}) \geq 0, \ldots, g_{s}(\bar{x}) \geq 0\right\} \subset \widetilde{\Delta}_{n}^{\circ}
$$

and such that the quadratic module $M\left(g_{1}, \ldots, g_{s}\right)$ is archimedean. There exists a positive constant $c$ such that for every $f \in \mathbb{R}[\bar{X}, Y]$ positive on $S \times \mathbb{R}$, if $\operatorname{deg}_{\bar{X}} f=d$, $\operatorname{deg}_{Y} f=m$ with $f$ fully $m$-ic on $S$ and

$$
\min \{\bar{f}(\bar{x}, y, z) \mid \bar{x} \in S,(y, z) \in C\}=f^{\bullet}>0
$$

$f$ can be written as

$$
f=\sigma_{0}+\sigma_{1} g_{1}+\cdots+\sigma_{s} g_{s} \in M_{\mathbb{R}[\bar{X}, Y]}\left(g_{1}, \ldots, g_{s}\right)
$$

with $\sigma_{0}, \sigma_{1}, \ldots, \sigma_{s} \in \sum \mathbb{R}[\bar{X}, Y]^{2}$ and

$$
\operatorname{deg}\left(\sigma_{0}\right), \operatorname{deg}\left(\sigma_{1} g_{1}\right), \ldots, \operatorname{deg}\left(\sigma_{s} g_{s}\right) \leq c(m+1) 2^{\frac{m}{2}} \mathrm{e}^{\left(\frac{\|f\| \bullet(m+1) d^{2}}{f^{\bullet}}\right)^{c}} .
$$

A nice fact about the bound in Proposition 10 is that it is singly exponential in $d$, meanwhile the bound in Theorem 7 is doubly exponential in $d$. This could be of independent interest even in the case $m=0$, this is to say, $f \in \mathbb{R}[X]$ positive on $S$.

\section{Proof of the main result}

As said in the introduction, to prove Theorem 7 we borrow and combine many ideas and techniques from [8], [9] and [13]. We start this section by quoting some results from these papers. We will also use many other general ideas from these sources which is not possible to quote independently but we want to give them credit for.

The following two auxiliary results come from [8, Remark 12] and [8, Proposition 14].

Remark 11 For every $k \in \mathbb{N}$ and $t \in[0,1]$,

$$
t \cdot(t-1)^{2 k} \leq \frac{1}{2 k+1} .
$$


Proposition 12 Let $g_{1}, \ldots, g_{s} \in \mathbb{R}[\bar{X}] \backslash\{0\}$. Then

$$
\left\|g_{1} \ldots g_{s}\right\| \leq\left(\operatorname{deg} g_{1}+1\right) \ldots\left(\operatorname{deg} g_{s}+1\right)\left\|g_{1}\right\| \ldots\left\|g_{s}\right\| .
$$

If in addition $g_{1}, \ldots, g_{s}$ are homogeneous, then

$$
\left\|g_{1} \ldots g_{s}\right\| \leq\left\|g_{1}\right\| \ldots\left\|g_{s}\right\|
$$

Next remark is present in the proof of [ 8 , Lemma 13] and is similar to a remark made in the proof of [13, Lemma 9].

Remark 13 Let $g_{1}, \ldots, g_{s} \in \mathbb{R}[\bar{X}]$ such that

$$
\emptyset \neq S=\left\{\bar{x} \in \mathbb{R}^{n} \mid g_{1}(\bar{x}) \geq 0, \ldots, g_{s}(\bar{x}) \geq 0\right\} \subset \widetilde{\Delta}_{n}^{\circ} .
$$

By Eojasiewicz inequality (see [1, Corollary 2.6.7]), there exist $c_{1}, c_{2}>0$ such that for every $\bar{x} \in \widetilde{\Delta}_{n}$,

$$
\operatorname{dist}(\bar{x}, S)^{c_{1}} \leq-c_{2} \min \left\{g_{1}(\bar{x}), \cdots, g_{s}(\bar{x}), 0\right\}
$$

In particular, for $x \in \widetilde{\Delta}_{n} \backslash S$, there exists $i_{0}$ with $1 \leq i_{0} \leq s$ such that $g_{i_{0}}(\bar{x})<0$ and

$$
\operatorname{dist}(\bar{x}, S)^{c_{1}} \leq-c_{2} g_{i_{0}}(\bar{x})
$$

The following remark will be useful in the proof of our main result and the idea of using a Putinar representation for a finite number of fixed polynomials comes from the proof of [13, Theorem 3].

Remark 14 Let $g_{1}, \ldots, g_{s} \in \mathbb{R}[\bar{X}]$ such that

$$
\emptyset \neq S=\left\{\bar{x} \in \mathbb{R}^{n} \mid g_{1}(\bar{x}) \geq 0, \ldots, g_{s}(\bar{x}) \geq 0\right\} \subset \widetilde{\Delta}_{n}^{\circ}
$$

and $M\left(g_{1}, \ldots, g_{s}\right)$ is archimedean. Since for each $v=\left(v_{0}, \bar{v}\right) \in\{0,1\}^{n+1}$

$$
\left(1-X_{1}-\cdots-X_{n}\right)^{v_{0}} \bar{X}^{\bar{v}}>0 \text { in } S
$$

by Putinar's Positivstellensatz ([11] ), there exist $\sigma_{v 0}, \sigma_{v 1}, \ldots, \sigma_{v s} \in \sum \mathbb{R}[\bar{X}]^{2}$ such that

$$
\left(1-X_{1}-\cdots-X_{n}\right)^{v_{0}} \bar{X}^{\bar{v}}=\sigma_{v 0}+\sigma_{v 1} g_{1}+\cdots+\sigma_{v s} g_{s}
$$

We need to extend the definition of \|\|$_{\bullet}$ to polynomials homogeneous in $(Y, Z)$ as follows.

Definition 15 For

$$
h=\sum_{0 \leq i \leq m} \sum_{\substack{\alpha \in \mathbb{N}_{0}^{n} \\
|\alpha| \leq d}}\left(\begin{array}{c}
|\alpha| \\
\alpha
\end{array}\right) a_{\alpha, i} \bar{X}^{\alpha} Y^{i} Z^{m-i} \in \mathbb{R}[\bar{X}, Y, Z]
$$

we consider the new norm defined also for $h$ by

$$
\|h\|_{\bullet}=\max \left\{\left|a_{\alpha, i}\right|\left|0 \leq i \leq m, \alpha \in \mathbb{N}_{0}^{n},\right| \alpha \mid \leq d\right\} .
$$


Note that $\|\bar{f}\|=\|f\|$ for every $f \in \mathbb{R}[\bar{X}, Y]$.

Next auxiliary lemma will be useful to prove the degree bound from Theorem [7, We use the notation $C=\left\{(y, z) \in \mathbb{R}^{2} \mid y^{2}+z^{2}=1\right\}$ which we introduced before and we keep for the rest of the paper.

Lemma 16 Let $f \in \mathbb{R}[\bar{X}, Y]$ such that $\operatorname{deg}_{\bar{X}} f=d$ and $\operatorname{deg}_{Y} f=m$. For every $\bar{x} \in \widetilde{\Delta}_{n}$, and $(y, z) \in C$,

$$
|\bar{f}(\bar{x}, y, z)| \leq\|f\|_{\bullet}(m+1)(d+1)
$$

Proof: Suppose

$$
f=\sum_{0 \leq i \leq m} \sum_{\substack{\alpha \in \mathbb{N}_{0}^{n} \\
|\alpha| \leq d}}\left(\begin{array}{c}
|\alpha| \\
\alpha
\end{array}\right) a_{\alpha, i} \bar{X}^{\alpha} Y^{i}
$$

Then

$$
\begin{aligned}
|\bar{f}(\bar{x}, y, z)| & \leq \sum_{0 \leq i \leq m} \sum_{\substack{\alpha \in \mathbb{N}_{0}^{n} \\
|\alpha| \leq d}}\left(\begin{array}{c}
|\alpha| \\
\alpha
\end{array}\right)\left|a_{\alpha, i}\right| \bar{x}^{\alpha}|y|^{i}|z|^{m-i} \\
& \leq\|f\| \cdot \sum_{\substack { 0 \leq i \leq m \\
\begin{subarray}{c}{\alpha \in \mathbb{N}_{0}^{n} \\
|\alpha| \leq d{ 0 \leq i \leq m \\
\begin{subarray} { c } { \alpha \in \mathbb { N } _ { 0 } ^ { n } \\
| \alpha | \leq d } }\end{subarray}}\left(\begin{array}{c}
|\alpha| \\
\alpha
\end{array}\right) \bar{x}^{\alpha} \\
& =\|f\| \cdot \sum_{0 \leq i \leq m} \sum_{0 \leq j \leq d} \sum_{\substack{\alpha \in \mathbb{N}_{0}^{n} \\
|\alpha|=j}}\left(\begin{array}{c}
|\alpha| \\
\alpha
\end{array}\right) \bar{x}^{\alpha} \\
& =\|f\|_{\bullet} \sum_{0 \leq i \leq m} \sum_{0 \leq j \leq d}\left(x_{1}+\cdots+x_{n}\right)^{j} \\
& \leq\|f\|_{\bullet} \sum_{0 \leq i \leq m} \sum_{0 \leq j \leq d} 1=\|f\|_{\bullet}(m+1)(d+1) .
\end{aligned}
$$

The following lemma is an adaptation from [8, Lemma 11].

Lemma 17 Let $f \in \mathbb{R}[\bar{X}, Y]$ such that $\operatorname{deg}_{\bar{X}} f=d$ and $\operatorname{deg}_{Y} f=m$. For every $\bar{x}_{1}, \bar{x}_{2} \in \widetilde{\Delta}_{n}$ and $(y, z) \in C$,

$$
\left|\bar{f}\left(\bar{x}_{1}, y, z\right)-\bar{f}\left(\bar{x}_{2}, y, z\right)\right| \leq \frac{1}{2} \sqrt{n}\|f\|_{\bullet}(m+1) d(d+1)\left\|\bar{x}_{1}-\bar{x}_{2}\right\|
$$

We include also a technical lemma similar to [ㅁ, Lemma 15].

Lemma 18 Given $\left(c_{1}, c_{2}, c_{3}, c_{4}, c_{5}, c_{6}\right) \in \mathbb{R}_{\geq 0}^{6}$, there exists a positive constant $c$ such that for every $r \in \mathbb{R}_{\geq 0}$,

$$
c_{1} r^{c_{2}} \leq c \mathrm{e}^{r^{c}} \quad \text { and } \quad c_{3} r^{c_{4}} \mathrm{e}^{c_{5} r^{c_{6}}} \leq c \mathrm{e}^{r^{c}}
$$

Before proving Proposition 10 we include some more notation. 
Notation 19 For $n \in \mathbb{N}$, we denote, as usual, by $\Delta_{n}$ the simplex

$$
\Delta_{n}=\left\{\left(x_{0}, \bar{x}\right) \in \mathbb{R}^{n+1} \mid \sum_{0 \leq i \leq n} x_{i}=1 \text { and } x_{i} \geq 0 \text { for } 0 \leq i \leq n\right\}
$$

We are ready to prove Proposition 10.

Proof of Proposition 10: Without loss of generality we suppose $\operatorname{deg} g_{i} \geq 1$ and $\left|g_{i}\right| \leq 1$ in $\widetilde{\Delta}_{n}$ for $1 \leq i \leq s$.

To prove the result, we take such a polynomial $f \in \mathbb{R}[\bar{X}, Y]$ and we need to show that we can find a constant $c$ which works independently from $f$. If $d=0$ then $f \in \mathbb{R}[Y]$ is positive on $\mathbb{R}$ and it is well-known that $f \in \sum \mathbb{R}[Y]^{2}$. Moreover, we can write $f$ as a sum of squares with the degree of each square bounded by $m$ (see [7, Proposition 1.2.1]), and then the degree bound simply holds for any constant $c \geq 1$. So from now we suppose $d \geq 1$ and if the final constant $c$ we find turns out to be less than 1 , we just replace it by the result of applying Lemma 18 to the 6 -uple $(1,0, c, 0,1, c)$. The new constant $c$ in particular satisfies for every $r \in \mathbb{R}_{\geq 0}$,

$$
1 \leq c \mathrm{e}^{r^{c}}
$$

and taking $r=0$ we have $c \geq 1$.

We prove first that there exist $\lambda \in \mathbb{R}_{>0}$ and $k \in \mathbb{N}_{0}$ such that

$$
h=\bar{f}-\lambda\left(Y^{2}+Z^{2}\right)^{\frac{m}{2}} \sum_{1 \leq i \leq s} g_{i} \cdot\left(g_{i}-1\right)^{2 k} \in \mathbb{R}[\bar{X}, Y, Z]
$$

satisfies $h \geq \frac{1}{2} f^{\bullet}$ in $\widetilde{\Delta}_{n} \times C$.

For each $(y, z) \in C$ we consider

$$
A_{y, z}=\left\{\bar{x} \in \widetilde{\Delta}_{n} \mid \bar{f}(\bar{x}, y, z) \leq \frac{3}{4} f^{\bullet}\right\}
$$

Note that $A_{y, z} \cap S=\emptyset$.

To exhibit sufficient conditions for $\lambda$ and $k$, we consider separately the cases $\bar{x} \in \widetilde{\Delta}_{n} \backslash A_{y, z}$ and $\bar{x} \in A_{y, z}$. If $\bar{x} \in \widetilde{\Delta}_{n} \backslash A_{y, z}$, by Remark [11,

$$
\begin{aligned}
h(\bar{x}, y, z) & =\bar{f}(\bar{x}, y, z)-\lambda\left(y^{2}+z^{2}\right)^{\frac{m}{2}} \sum_{1 \leq i \leq s} g_{i}(\bar{x}) \cdot\left(g_{i}(\bar{x})-1\right)^{2 k} \\
& \geq \bar{f}(\bar{x}, y, z)-\lambda \sum_{1 \leq i \leq s}\left|g_{i}(\bar{x})\right| \cdot\left(\left|g_{i}(\bar{x})\right|-1\right)^{2 k} \\
& >\frac{3}{4} f^{\bullet}-\frac{\lambda s}{2 k+1} .
\end{aligned}
$$

Therefore the condition $h(\bar{x}, y, z) \geq \frac{1}{2} f^{\bullet}$ is ensured if

$$
2 k+1 \geq \frac{4 \lambda s}{f^{\bullet}}
$$


If $\bar{x} \in A_{y, z}$, for any $\bar{x}_{0} \in S$, by Lemma 17 we have

$$
\frac{f^{\bullet}}{4} \leq \bar{f}\left(\bar{x}_{0}, y, z\right)-\bar{f}(\bar{x}, y, z) \leq \frac{1}{2} \sqrt{n}\|f\|_{\bullet}(m+1) d(d+1)\left\|\bar{x}_{0}-\bar{x}\right\|,
$$

then

$$
\frac{f^{\bullet}}{2 \sqrt{n}\|f\|_{\bullet}(m+1) d(d+1)} \leq\left\|\bar{x}_{0}-\bar{x}\right\|
$$

and therefore

$$
\frac{f^{\bullet}}{2 \sqrt{n}\|f\|_{\bullet}(m+1) d(d+1)} \leq \operatorname{dist}(\bar{x}, S) .
$$

By Remark 13, there exist $c_{1}, c_{2}>0$ and $1 \leq i_{0} \leq s$ such that $g_{i_{0}}(\bar{x})<0$ and

$$
\operatorname{dist}(\bar{x}, S)^{c_{1}} \leq-c_{2} g_{i_{0}}(\bar{x})
$$

By (3) and (4) we have

$$
g_{i_{0}}(\bar{x}) \leq-\delta
$$

with

$$
\delta=\frac{1}{c_{2}}\left(\frac{f^{\bullet}}{2 \sqrt{n}\|f\|_{\bullet}(m+1) d(d+1)}\right)^{c_{1}}>0
$$

On the other hand, defining $f_{y, z}^{\bullet}=\min \{\bar{f}(\bar{x}, y, z) \mid \bar{x} \in S\}$, again by Lemma 17 we have that

$$
\left|\bar{f}(\bar{x}, y, z)-f_{y, z}^{\bullet}\right| \leq \frac{1}{2} \sqrt{n}\|f\|_{\bullet}(m+1) d(d+1) \operatorname{diam}\left(\widetilde{\Delta}_{n}\right)=\frac{1}{\sqrt{2}} \sqrt{n}\|f\|_{\bullet}(m+1) d(d+1) .
$$

Then, by Remark [1] and using (51) and (6) we have

$$
\begin{aligned}
h(\bar{x}, y, z) & \geq \bar{f}(\bar{x}, y, z)-\lambda g_{i_{0}}(\bar{x})\left(g_{i_{0}}(\bar{x})-1\right)^{2 k}-\frac{\lambda(s-1)}{2 k+1} \\
& \geq \bar{f}(\bar{x}, y, z)-f_{y, z}^{\bullet}+f_{y, z}^{\bullet}+\lambda \delta-\frac{\lambda(s-1)}{2 k+1} \\
& \geq-\frac{1}{\sqrt{2}} \sqrt{n}\|f\|_{\bullet}(m+1) d(d+1)+f^{\bullet}+\lambda \delta-\frac{\lambda(s-1)}{2 k+1} .
\end{aligned}
$$

Finally, the condition $h(\bar{x}, y, z) \geq \frac{1}{2} f^{\bullet}$ is ensured if

$$
\lambda \geq \frac{\sqrt{n}\|f\|_{\bullet}(m+1) d(d+1)}{\sqrt{2} \delta}=\frac{c_{2} 2^{c_{1}}\left(\sqrt{n}\|f\|_{\bullet}(m+1) d(d+1)\right)^{c_{1}+1}}{\sqrt{2} f^{\bullet c_{1}}}
$$

and

$$
2 k+1 \geq \frac{2 \lambda(s-1)}{f^{\bullet}} .
$$

Since (2) implies (8), it is enough for $\lambda$ and $k$ to satisfy (22) and (7). So for the rest of the proof we take

$$
\lambda=\frac{c_{2} 2^{c_{1}}\left(\sqrt{n}\|f\|_{\bullet}(m+1) d(d+1)\right)^{c_{1}+1}}{\sqrt{2} f^{\bullet c_{1}}}=c_{3} \frac{\left(\|f\|_{\bullet}(m+1) d(d+1)\right)^{c_{1}+1}}{f^{\bullet c_{1}}}>0
$$


with $c_{3}=\frac{c_{2} 2^{c_{1}} \sqrt{n}^{c_{1}+1}}{\sqrt{2}}$ and

$$
k=\left\lceil\frac{1}{2}\left(\frac{4 \lambda s}{f^{\bullet}}-1\right)\right\rceil \in \mathbb{N}_{0}
$$

In this way,

$$
\begin{aligned}
k & \leq \frac{1}{2}\left(\frac{4 \lambda s}{f^{\bullet}}-1\right)+1 \\
& =2 c_{3} s\left(\frac{\|f\|_{\bullet}(m+1) d(d+1)}{f^{\bullet}}\right)^{c_{1}+1}+\frac{1}{2} \\
& \leq c_{4}\left(\frac{\|f\|_{\bullet}(m+1) d(d+1)}{f^{\bullet}}\right)^{c_{1}+1}
\end{aligned}
$$

with $c_{4}=2 c_{3} s+1$. Here (and also several times after here) we use Lemma 16] to ensure

$$
\frac{\|f\|_{\bullet}(m+1)(d+1)}{f^{\bullet}} \geq 1 .
$$

Also, if we define $\ell=\operatorname{deg}_{\bar{X}} h$, we have

$$
\begin{aligned}
\ell & \leq \max \left\{d,(2 k+1) \max _{1 \leq i \leq s} \operatorname{deg} g_{i}\right\} \\
& \leq \max \left\{d,\left(2 c_{4}\left(\frac{\|f\|_{\bullet}(m+1) d(d+1)}{f^{\bullet}}\right)^{c_{1}+1}+1\right) \max _{1 \leq i \leq s} \operatorname{deg} g_{i}\right\} \\
& \leq c_{5}\left(\frac{\|f\|_{\bullet}(m+1) d(d+1)}{f^{\bullet}}\right)^{c_{1}+1}
\end{aligned}
$$

with $c_{5}=\left(2 c_{4}+1\right) \max _{1 \leq i \leq s} \operatorname{deg} g_{i}$.

On the other hand, using Proposition 12 and (9),

$$
\begin{aligned}
\|h\|_{\bullet} \leq & \|f\|_{\bullet}+\lambda s 2^{\frac{m}{2}} \max _{1 \leq i \leq s}\left\{\left(\operatorname{deg} g_{i}+1\right)\left(\left\|g_{i}\right\|+1\right)\right\}^{2 k+1} \\
= & \|f\|_{\bullet}+c_{3} s 2^{\frac{m}{2}} \frac{\left(\|f\|_{\bullet}(m+1) d(d+1)\right)^{c_{1}+1}}{f^{\bullet c_{1}}} \max _{1 \leq i \leq s}\left\{\left(\operatorname{deg} g_{i}+1\right)\left(\left\|g_{i}\right\|+1\right)\right\}^{2 k+1} \\
\leq & \left(c_{3} s+1\right) \max _{1 \leq i \leq s}\left\{\left(\operatorname{deg} g_{i}+1\right)\left(\left\|g_{i}\right\|+1\right)\right\} \cdot \\
& \cdot 2^{\frac{m}{2}} \frac{\left(\|f\|_{\bullet}(m+1) d(d+1)\right)^{c_{1}+1}}{f^{\bullet c_{1}}} \max _{1 \leq i \leq s}\left\{\left(\operatorname{deg} g_{i}+1\right)\left(\left\|g_{i}\right\|+1\right)\right\}^{2 c_{4}\left(\frac{\|f\| \bullet(m+1) d(d+1)}{f^{\bullet}}\right)^{c_{1}+1}} \\
= & c_{6} 2^{\frac{m}{2}} \frac{\left(\|f\|_{\bullet}(m+1) d(d+1)\right)^{c_{1}+1}}{f^{\bullet} c_{1}} \mathrm{e}^{c_{7}\left(\frac{\|f\| \bullet(m+1) d(d+1)}{f^{\bullet}}\right)^{c_{1}+1}}
\end{aligned}
$$

with $c_{6}=\left(c_{3} s+1\right) \max _{1 \leq i \leq s}\left\{\left(\operatorname{deg} g_{i}+1\right)\left(\left\|g_{i}\right\|+1\right)\right\}$ and $c_{7}=\log \left(\max _{1 \leq i \leq s}\left\{\left(\operatorname{deg} g_{i}+1\right)\left(\left\|g_{i}\right\|+1\right)\right\}^{2 c_{4}}\right)$.

So far we have found $\lambda$ and $k$ such that that $h \geq \frac{1}{2} f^{\bullet}$ in $\widetilde{\Delta}_{n} \times C$, together with bounds for $k, \ell=\operatorname{deg}_{\bar{X}} h$ and $\|h\|_{\bullet}$. Now, we introduce a new variable $X_{0}$ with the aim of homogenize with respect to the 
variables $\bar{X}$ and be able to use Pólya's Theorem. Let

$$
h=\sum_{0 \leq i \leq m} \sum_{0 \leq j \leq \ell} h_{i j}(\bar{X}) Y^{i} Z^{m-i}
$$

with $h_{i j} \in \mathbb{R}[\bar{X}]$ equal to zero or homogeneous of degree $j$ for $0 \leq i \leq m$ and $0 \leq j \leq \ell$. We define

$$
H=\sum_{0 \leq i \leq m} \sum_{0 \leq j \leq \ell} h_{i j}(\bar{X})\left(X_{0}+X_{1} \cdots+X_{n}\right)^{\ell-j} Y^{i} Z^{m-i} \in \mathbb{R}\left[X_{0}, \bar{X}, Y, Z\right]
$$

which is bihomogeneous in $\left(X_{0}, \bar{X}\right)$ and $(Y, Z)$ (i.e. homogeneous in the variables $\left(X_{0}, \bar{X}\right)$ and $(Y, Z)$ separately).

Since $H\left(x_{0}, \bar{x}, y, z\right)=h(\bar{x}, y, z)$ for every $\left(x_{0}, \bar{x}, y, z\right) \in \Delta_{n} \times C$, it is clear that $H \geq \frac{1}{2} f^{\bullet}$ in $\Delta_{n} \times C$.

On the other hand, for each $(y, z) \in C$, we consider $H\left(X_{0}, X, y, z\right) \in \mathbb{R}\left[X_{0}, \bar{X}\right]$. Using Proposition 12 we have

$$
\begin{aligned}
\left\|H\left(X_{0}, X, y, z\right)\right\| & \leq \sum_{0 \leq i \leq m} \sum_{0 \leq j \leq \ell}\left\|h_{i j}(\bar{X})\left(X_{0}+\cdots+X_{n}\right)^{\ell-j} y^{i} z^{m-i}\right\| \\
& \leq \sum_{0 \leq i \leq m} \sum_{0 \leq j \leq \ell}\left\|h_{i j}(\bar{X})\left(X_{0}+\cdots+X_{n}\right)^{\ell-j}\right\| \\
& \leq \sum_{0 \leq i \leq m} \sum_{0 \leq j \leq \ell}\left\|h_{i j}(\bar{X})\right\| \\
& \leq(m+1)(\ell+1)\|h\|_{\bullet} .
\end{aligned}
$$

We use now the bound for Pólya's Theorem from [10, Theorem 1]. Take $N \in \mathbb{N}$ given by

$$
N=\left\lfloor\frac{(m+1)(\ell+1) \ell(\ell-1)\|h\|_{\bullet}}{f^{\bullet}}-\ell\right\rfloor+1 .
$$

Then for each $(y, z) \in C$ we have that $H\left(X_{0}, \bar{X}, y, z\right)\left(X_{0}+X_{1}+\cdots+X_{n}\right)^{N} \in \mathbb{R}\left[X_{0}, \bar{X}\right]$ is a homogeneous polynomial such that all its coefficients are positive. More precisely, suppose we write

$$
H\left(X_{0}, \bar{X}, Y, Z\right)\left(X_{0}+X_{1}+\cdots+X_{n}\right)^{N}=\sum_{\substack{\alpha=\left(\alpha_{0}, \bar{\alpha}\right) \in \mathbb{N}_{0}^{n+1} \\|\alpha|=N+\ell}} b_{\alpha}(Y, Z) X_{0}^{\alpha_{0}} \bar{X}^{\bar{\alpha}} \in \mathbb{R}\left[X_{0}, \bar{X}, Y, Z\right]
$$

with $b_{\alpha} \in \mathbb{R}[Y, Z]$ homogeneous of degree $m$. The conclusion is that for every $\alpha \in \mathbb{N}_{0}^{n+1}$ with $|\alpha|=$ $N+\ell$, the polynomial $b_{\alpha}$ is positive in $C$, and therefore, since it is a homogenous polynomial, $b_{\alpha}$ is non-negative in $\mathbb{R}^{2}$.

Before going on, we bound $N+\ell$ using (10) and (11) as follows.

$$
\begin{aligned}
N+\ell & \leq \frac{(m+1)(\ell+1) \ell(\ell-1)\|h\|_{\bullet}}{f^{\bullet}}+1 \\
& \leq \frac{(m+1) \ell^{3}\|h\|_{\bullet}}{f^{\bullet}}+1 \\
& \leq c_{5}^{3} c_{6}(m+1) 2^{\frac{m}{2}}\left(\frac{\|f\|_{\bullet}(m+1) d(d+1)}{f^{\bullet}}\right)^{4\left(c_{1}+1\right)} \mathrm{e}^{c_{7}\left(\frac{\|f\|_{\bullet}(m+1) d(d+1)}{f^{\bullet}}\right)^{c_{1}+1}}+1 \\
& \leq c_{8}(m+1) 2^{\frac{m}{2}}\left(\frac{\|f\|_{\bullet}(m+1) d(d+1)}{f^{\bullet}}\right)^{4\left(c_{1}+1\right)} \mathrm{e}^{c_{7}\left(\frac{\|f\|_{\bullet}(m+1) d(d+1)}{f^{\bullet}}\right)^{c_{1}+1}}
\end{aligned}
$$


with $c_{8}=c_{5}^{3} c_{6}+1$.

Now we substitute $X_{0}=1-X_{1}-\cdots-X_{n}$ and $Z=1$ in (12) and we obtain

$$
f=\lambda\left(Y^{2}+1\right)^{\frac{m}{2}} \sum_{1 \leq i \leq s} g_{i}\left(g_{i}-1\right)^{2 k}+\sum_{\substack{\alpha=\left(\alpha_{0}, \bar{\alpha}\right) \in \mathbb{N}_{0}^{n+1} \\|\alpha|=N+\ell}} b_{\alpha}(Y, 1)\left(1-X_{1}-\cdots-X_{n}\right)^{\alpha_{0}} \bar{X}^{\bar{\alpha}} \in \mathbb{R}[\bar{X}, Y]
$$

From (14) we want to conclude that $f \in M_{\mathbb{R}[\bar{X}, Y]}\left(g_{1} \ldots, g_{s}\right)$ and to find the positive constant $c$ such that the degree bound holds.

The first term on the right hand side of (14) clearly belongs to $M_{\mathbb{R}[\bar{X}, Y]}\left(g_{1} \ldots, g_{s}\right)$. Moreover, for $1 \leq i \leq s$,

$$
\operatorname{deg}\left(Y^{2}+1\right)^{\frac{m}{2}} g_{i}\left(g_{i}-1\right)^{2 k} \leq m+(2 k+1) \operatorname{deg} g_{i}
$$

Now we focus on the second term on the right hand side of (14), which is itself a sum. Take a fixed $\alpha \in \mathbb{N}_{0}^{n+1}$ with $|\alpha|=N+\ell$.

Since $b_{\alpha}(Y, 1)$ is non-negative in $\mathbb{R}, b_{\alpha}(Y, 1) \in \sum \mathbb{R}[Y]^{2}$. Moreover, we can write $b_{\alpha}(Y, 1)$ as a sum of squares with the degree of each square bounded by $m$ (see [7, Proposition 1.2.1]).

Also, take $v(\alpha)=\left(v_{0}, \bar{v}\right) \in\{0,1\}^{n+1}$ such that $\alpha_{i} \equiv v_{i}(\bmod 2)$ for $0 \leq i \leq n$. Denoting $g_{0}=1 \in \mathbb{R}[\bar{X}]$, by Remark 14, we have

$$
\left(1-X_{1}-\cdots-X_{n}\right)^{v_{0}} \bar{X}^{\bar{v}}=\sum_{0 \leq i \leq s} \sigma_{v(\alpha) i} g_{i},
$$

with $\sigma_{v(\alpha) i} \in \sum \mathbb{R}[\bar{X}]^{2}$ for $0 \leq i \leq s$, and then

$$
\left(1-X_{1}-\cdots-X_{n}\right)^{\alpha_{0}} \bar{X}^{\bar{\alpha}}=\left(1-X_{1}-\cdots-X_{n}\right)^{\alpha_{0}-v_{0}} \bar{X}^{\bar{\alpha}-\bar{v}} \sum_{0 \leq i \leq s} \sigma_{v(\alpha) i} g_{i}
$$

belongs to $M\left(g_{1}, \ldots, g_{s}\right)$ since $\left(1-X_{1}-\cdots-X_{n}\right)^{\alpha_{0}-v_{0}} \bar{X}^{\bar{\alpha}-\bar{v}} \in \mathbb{R}[\bar{X}]^{2}$.

We conclude in this way that each term in the sum belongs to $M_{\mathbb{R}[\bar{X}, Y]}\left(g_{1}, \ldots, g_{s}\right)$. In addition, for $0 \leq i \leq s$ we have

$$
\operatorname{deg} b_{\alpha}(Y, 1)\left(1-X_{1}-\cdots-X_{n}\right)^{\alpha_{0}-v_{0}} \bar{X}^{\bar{\alpha}-\bar{v}} \sigma_{v(\alpha) i} g_{i} \leq m+N+\ell+c_{9}
$$

with $c_{9}=\max \left\{\operatorname{deg} \sigma_{v i} g_{i} \mid v \in\{0,1\}^{n+1}, 0 \leq i \leq s\right\}$.

To finish the proof, we only need to bound simultaneously the right hand side of (15) and (16).

On the one hand, using (9),

$$
\begin{aligned}
m+(2 k+1) \max _{1 \leq i \leq s} \operatorname{deg} g_{i} & \leq m+\left(2 c_{4}\left(\frac{\|\bar{f}\|_{\bullet}(m+1) d(d+1)}{f^{\bullet}}\right)^{c_{1}+1}+1\right) \max _{1 \leq i \leq s} \operatorname{deg} g_{i} \\
& \leq c_{10}(m+1)\left(\frac{\|\bar{f}\|_{\bullet}(m+1) d^{2}}{f^{\bullet}}\right)^{c_{1}+1}
\end{aligned}
$$

with $c_{10}=\left(2 c_{4}+1\right) 2^{c_{1}+1} \max _{1 \leq i \leq s} \operatorname{deg} g_{i}$, since $d \geq 1$. 
On the other hand, using (13),

$$
\begin{aligned}
m+N+\ell+c_{9} & \leq m+c_{8}(m+1) 2^{\frac{m}{2}}\left(\frac{\|f\| \bullet(m+1) d(d+1)}{f^{\bullet}}\right)^{4\left(c_{1}+1\right)} \mathrm{e}^{c_{7}\left(\frac{\|\bar{f}\| \bullet(m+1) d(d+1)}{f^{\bullet}}\right)^{c_{1}+1}}+c_{9} \\
& \leq c_{11}(m+1) 2^{\frac{m}{2}}\left(\frac{\|f\| \bullet(m+1) d^{2}}{f^{\bullet}}\right)^{4\left(c_{1}+1\right)} \mathrm{e}^{c_{12}\left(\frac{\|f\| \bullet(m+1) d^{2}}{f^{\bullet}}\right)^{c_{1}+1}}
\end{aligned}
$$

with $c_{11}=\left(1+c_{8}+c_{9}\right) 2^{4\left(c_{1}+1\right)}$ and $c_{12}=c_{7} 2^{c_{1}+1}$, again since $d \geq 1$.

Finally, we define $c$ as the positive constant obtained applying Lemma 18 to the 6 -uple $\left(c_{10}, c_{1}+1\right.$, $\left.c_{11}, 4\left(c_{1}+1\right), c_{12}, c_{1}+1\right)$.

Before going to the proof of our main result, we include a technical lemma with a bound.

Lemma 20 Let $j, d \in \mathbb{N}_{0}$ with $j \leq d$. Then

$$
2^{j}\left(\begin{array}{c}
d+1 \\
j+1
\end{array}\right) \leq 3^{d}
$$

Proof: We proceed by induction on $d$. For $d=0$ we check the inequality by hand. Now suppose $d \geq 1$. If $j=0$ or $j=d$ again we check the inequality by hand. If $1 \leq j \leq d-1$ then

$$
2^{j}\left(\begin{array}{l}
d+1 \\
j+1
\end{array}\right)=2 \cdot 2^{j-1}\left(\begin{array}{l}
d \\
j
\end{array}\right)+2^{j}\left(\begin{array}{c}
d \\
j+1
\end{array}\right) \leq 2 \cdot 3^{d-1}+3^{d-1}=3^{d} .
$$

We are ready to prove Theorem 7 .

Proof of Theorem 7 ; We consider the affine change of variables $\ell: \mathbb{R}^{n} \rightarrow \mathbb{R}^{n}$ given by

$$
\ell\left(X_{1}, \ldots, X_{n}\right)=\left(\frac{X_{1}+1}{2 n}, \ldots, \frac{X_{n}+1}{2 n}\right) .
$$

For $0 \leq i \leq s$, we take $\tilde{g}_{i}(\bar{X})=g_{i}\left(\ell^{-1}(\bar{X})\right) \in \mathbb{R}[\bar{X}]$ and we define

$$
\widetilde{S}=\left\{\bar{x} \in \mathbb{R}^{n} \mid \tilde{g}_{1}(\bar{x}) \geq 0, \ldots, \tilde{g}_{s}(\bar{x}) \geq 0\right\} .
$$

It is easy to see that

$$
\emptyset \neq \widetilde{S}=\ell(S) \subseteq \widetilde{\Delta}_{n}^{\circ}
$$

Moreover, since $M\left(g_{1}, \ldots, g_{n}\right)$ is archimedean, $M\left(\tilde{g}_{1}, \ldots, \tilde{g}_{s}\right)$ is also archimedean. To see this, following the proof of [7, Proposition 5.2.3], if

$$
N-X_{1}^{2}-\cdots-X_{n}^{2} \in M\left(g_{1}, \ldots, g_{n}\right),
$$

then

$$
\frac{N}{2 n^{2}}+\frac{1}{2 n}-\left(\frac{X_{1}+1}{2 n}\right)^{2}-\cdots-\left(\frac{X_{n}+1}{2 n}\right)^{2}=
$$




$$
=\frac{1}{2 n^{2}}\left(N-X_{1}^{2}-\cdots-X_{n}^{2}\right)+\frac{1}{4 n^{2}}\left(\left(X_{1}-1\right)^{2}+\cdots+\left(X_{n}-1\right)^{2}\right) \in M\left(g_{1}, \ldots, g_{n}\right)
$$

and composing with $\ell^{-1}$ we have

$$
\frac{N}{2 n^{2}}+\frac{1}{2 n}-X_{1}^{2}-\cdots-X_{n}^{2} \in M\left(\tilde{g}_{1}, \ldots, \tilde{g}_{n}\right) .
$$

Let $f \in \mathbb{R}[\bar{X}, Y]$ be as in the statement of Theorem 7 and let $\tilde{f}(\bar{X}, Y)=f\left(\ell^{-1}(\bar{X}), Y\right) \in \mathbb{R}[\bar{X}, Y]$. It can be easily seen that $\tilde{f}$ is positive on $\tilde{S} \times \mathbb{R}, \operatorname{deg}_{\bar{X}} \tilde{f}=\operatorname{deg}_{\bar{X}} f=d, \operatorname{deg}_{Y} \tilde{f}=\operatorname{deg}_{Y} f=m, \tilde{f}$ is fully $m$-ic on $\widetilde{S}$ and

$$
\min \{\overline{\tilde{f}}(\bar{x}, y, z) \mid \bar{x} \in \widetilde{S},(y, z) \in C\}=\min \{\bar{f}(\bar{x}, y, z) \mid \bar{x} \in S,(y, z) \in C\}=f^{\bullet}>0,
$$

Now we want to bound $\|\tilde{f}\|_{\bullet}$. So suppose

$$
f=\sum_{0 \leq i \leq m} \sum_{\substack{\alpha \in \mathbb{N}_{0}^{n} \\
|\alpha| \leq d}}\left(\begin{array}{c}
|\alpha| \\
\alpha
\end{array}\right) a_{\alpha, i} \bar{X}^{\alpha} Y^{i}
$$

Then

$$
\begin{aligned}
\tilde{f} & =\sum_{\substack { 0 \leq i \leq m \\
\begin{subarray}{c}{\alpha \in \mathbb{N}_{0}^{n} \\
|\alpha| \leq d{ 0 \leq i \leq m \\
\begin{subarray} { c } { \alpha \in \mathbb { N } _ { 0 } ^ { n } \\
| \alpha | \leq d } }\end{subarray}}\left(\begin{array}{c}
|\alpha| \\
\alpha
\end{array}\right) a_{\alpha, i}\left(2 n X_{1}-1, \cdots, 2 n X_{n}-1\right)^{\alpha} Y^{i} \\
& =\sum_{\substack { 0 \leq i \leq m \\
\begin{subarray}{c}{\alpha \in \mathbb{N}_{0}^{n} \\
|\alpha| \leq d{ 0 \leq i \leq m \\
\begin{subarray} { c } { \alpha \in \mathbb { N } _ { 0 } ^ { n } \\
| \alpha | \leq d } }\end{subarray}}\left(\begin{array}{c}
|\alpha| \\
\alpha
\end{array}\right) a_{\alpha, i} \sum_{\substack{\beta \in \mathbb{N}_{0}^{n} \\
\beta \preceq \alpha}}\left(\begin{array}{c}
\alpha_{1} \\
\beta_{1}
\end{array}\right) \cdots\left(\begin{array}{c}
\alpha_{n} \\
\beta_{n}
\end{array}\right)(2 n)^{|\beta|}(-1)^{|\alpha|-|\beta|} \bar{X}^{\beta} Y^{i} \\
& =\sum_{0 \leq i \leq m} \sum_{\substack{\beta \in \mathbb{N}_{0}^{n} \\
|\beta| \leq d}}(2 n)^{|\beta|} \sum_{\substack{\alpha \in \mathbb{N}_{0}^{n} \\
\beta \preceq \alpha,|\alpha| \leq d}}\left(\begin{array}{c}
|\alpha| \\
\alpha
\end{array}\right)\left(\begin{array}{c}
\alpha_{1} \\
\beta_{1}
\end{array}\right) \cdots\left(\begin{array}{c}
\alpha_{n} \\
\beta_{n}
\end{array}\right) a_{\alpha, i}(-1)^{|\alpha|-|\beta|} \bar{X}^{\beta} Y^{i} \\
& =\sum_{0 \leq i \leq m} \sum_{\substack{\beta \in \mathbb{N}_{0}^{n} \\
|\beta| \leq d}}\left(\begin{array}{c}
|\beta| \\
\beta
\end{array}\right)(2 n)^{|\beta|} \frac{1}{|\beta| !} \sum_{\substack{\alpha \in \mathbb{N}_{0}^{n} \\
\beta \preceq \alpha,|\alpha| \leq d}} \frac{|\alpha| !}{\left(\alpha_{1}-\beta_{1}\right) ! \cdots\left(\alpha_{n}-\beta_{n}\right) !} a_{\alpha, i}(-1)^{|\alpha|-|\beta|} \bar{X}^{\beta} Y^{i}
\end{aligned}
$$

For $0 \leq i \leq m$ and $\beta \in \mathbb{N}_{0}^{n}$ with $|\beta| \leq d$ we define

$$
b_{\beta, i}=(2 n)^{|\beta|} \frac{1}{|\beta| !} \sum_{\substack{\alpha \in \mathbb{N}_{0}^{n} \\ \beta \preceq \alpha,|\alpha| \leq d}} \frac{|\alpha| !}{\left(\alpha_{1}-\beta_{1}\right) ! \cdots\left(\alpha_{n}-\beta_{n}\right) !} a_{\alpha, i}(-1)^{|\alpha|-|\beta|} \in \mathbb{R}
$$


Then we have

$$
\begin{aligned}
& \left|b_{\beta, i}\right| \leq\|f\|_{\bullet}(2 n)^{|\beta|} \frac{1}{|\beta| !} \sum_{\substack{\alpha \in \mathbb{N}_{0}^{n} \\
\beta \preceq \alpha,|\alpha| \leq d}} \frac{|\alpha| !}{\left(\alpha_{1}-\beta_{1}\right) ! \cdots\left(\alpha_{n}-\beta_{n}\right) !} \\
& =\|f\|_{\bullet}(2 n)^{|\beta|} \frac{1}{|\beta| !} \sum_{|\beta| \leq h \leq d} h ! \sum_{\substack{\alpha \in \mathbb{N}_{0}^{n} \\
\beta \preceq \alpha,|\alpha|=h}} \frac{1}{\left(\alpha_{1}-\beta_{1}\right) ! \cdots\left(\alpha_{n}-\beta_{n}\right) !} \\
& =\|f\|_{\bullet}(2 n)^{|\beta|} \frac{1}{|\beta| !} \sum_{|\beta| \leq h \leq d} \frac{h !}{(h-|\beta|) !} \sum_{\substack{\gamma \in \mathbb{N}_{0}^{n} \\
|\gamma|=h-|\beta|}}\left(\begin{array}{c}
|\gamma| \\
\gamma
\end{array}\right) \\
& =\|f\|_{\bullet}(2 n)^{|\beta|} \sum_{|\beta| \leq h \leq d}\left(\begin{array}{c}
h \\
|\beta|
\end{array}\right) n^{h-|\beta|} \\
& \leq\|f\|_{\bullet} 2^{|\beta|} n^{d} \sum_{|\beta| \leq h \leq d}\left(\begin{array}{c}
h \\
|\beta|
\end{array}\right) \\
& =\|f\|_{\bullet} 2^{|\beta|} n^{d}\left(\begin{array}{c}
d+1 \\
|\beta|+1
\end{array}\right) \\
& \leq\|f\|_{\bullet}(3 n)^{d}
\end{aligned}
$$

using Lemma 20, So the conclusion is $\|\tilde{f}\|_{\bullet} \leq\|f\|_{\bullet}(3 n)^{d}$.

Finally, take $c$ as the positive constant from Proposition 10 applied to $\tilde{g}_{1}, \ldots, \tilde{g}_{s}$. Therefore, $\tilde{f}$ can be written as

$$
\tilde{f}=\tilde{\sigma}_{0}+\tilde{\sigma}_{1} \tilde{g}_{1}+\cdots+\tilde{\sigma}_{s} \tilde{g}_{s} \in M_{\mathbb{R}[\bar{X}, Y]}\left(\tilde{g}_{1}, \ldots, \tilde{g}_{s}\right)
$$

with $\tilde{\sigma}_{0}, \tilde{\sigma}_{1}, \ldots, \tilde{\sigma}_{s} \in \sum \mathbb{R}[\bar{X}, Y]^{2}$ and

$$
\operatorname{deg}\left(\tilde{\sigma}_{0}\right), \operatorname{deg}\left(\tilde{\sigma}_{1} \tilde{g}_{1}\right), \ldots, \operatorname{deg}\left(\tilde{\sigma}_{s} \tilde{g}_{s}\right) \leq c(m+1) 2^{\frac{m}{2}} \mathrm{e}^{\left(\frac{\|f\| \bullet(m+1) d^{2}(3 n) d}{f^{\bullet}}\right)^{c}}
$$

and the desired representation for $f$ is simply obtained by composing with $\ell$.

Remark 21 If $n \geq 2$, the factor $3^{d}$ in the exponentiation in the bound from Theorem 7 can be hidden in the constant $c$ as follows. If $d=0$, as explained before, the degree bound holds for any $c \geq 1$. If $d \geq 1$, then, using Lemma 16 ,

$$
\begin{gathered}
\frac{\|f\|_{\bullet}(m+1) d^{2}(3 n)^{d}}{f^{\bullet}} \leq \frac{\|f\|_{\bullet}(m+1) d(d+1)(3 n)^{d}}{f^{\bullet}} \leq \\
\leq\left(\frac{\|f\|_{\bullet}(m+1) d(d+1) n^{d}}{f^{\bullet}}\right)^{3} \leq 8\left(\frac{\|f\|_{\bullet}(m+1) d^{2} n^{d}}{f^{\bullet}}\right)^{3} .
\end{gathered}
$$

So we replace $c$ by the result of applying Lemma 18 to the 6 -uple $\left(1,0, c, 0,8^{c}, 3 c\right)$ and we are done.

Acknowledgements: We are thankful to the reviewers for their helpful suggestions and remarks. 


\section{References}

[1] J. Bochnak, M. Coste, M.-F. Roy, Real algebraic geometry. Ergebnisse der Mathematik und ihrer Grenzgebiete 36, Springer, Berlin, 1998.

[2] E. de Klerk, M. Laurent, Error bounds for some semidefinite programming approaches to polynomial minimization on the hypercube. SIAM J. Optim. 20 (2010), no. 6, 3104-3120.

[3] T. Jacobi, A. Prestel, Distinguished representations of strictly positive polynomials. J. Reine Angew. Math. 532 (2001), 223-235.

[4] S. Kuhlmann, M. Marshall, Positivity, sums of squares and the multi-dimensional moment problem. Trans. Amer. Math. Soc. 354 (2002), no. 11, 4285-4301.

[5] S. Kuhlmann, M. Marshall, N. Schwartz, Positivity, sums of squares and the multi-dimensional moment problem. II. Adv. Geom. 5 (2005), no. 4, 583-606.

[6] V. Magron, Error bounds for polynomial optimization over the hypercube using Putinar type representations. Optim. Lett. 9 (2015), no. 5, 887-895.

[7] M. Marshall, Positive polynomials and sums of squares. Mathematical Surveys and Monographs, 146. American Mathematical Society, Providence, RI, 2008.

[8] J. Nie, M. Schweighofer, On the complexity of Putinar's Positivstellensatz. J. Complexity 23 (2007), no. 1, 135-150.

[9] V. Powers, Positive polynomials and the moment problem for cylinders with compact crosssection. J. Pure Appl. Algebra 188 (2004), no. 1-3, 217-226.

[10] V. Powers, B. Reznick, A new bound for Pólya's theorem with applications to polynomials positive on polyhedra. J. Pure Appl. Algebra 164 (2001), no. 1-2, 221-229.

[11] M. Putinar, Positive polynomials on compact semi-algebraic sets. Indiana Univ. Math. J. 42 (1993), no. 3, 969-984.

[12] K. Schmüdgen, The K-moment problem for compact semi-algebraic sets. Math. Ann. 289 (1991), no. 2, 203-206.

[13] M. Schweighofer, On the complexity of Schmüdgen's Positivstellensatz. J. Complexity 20 (2004), no. $4,529-543$.

[14] G. Stengle, Complexity estimates for the Schmüdgen Positivstellensatz. J. Complexity 12 (1996), no. $2,167-174$. 